\title{
Affective regulation through touch: homeostatic and allostatic mechanisms Aikaterini Fotopoulou ${ }^{1}$, Mariana von Mohr $^{2}$ and Charlotte Krahé ${ }^{3}$
}

We focus on social touch as a paradigmatic case of the embodied, cognitive, and metacognitive processes involved in social, affective regulation. Social touch appears to contribute three interrelated but distinct functions to affective regulation. First, it regulates affects by fulfilling embodied predictions about social proximity and attachment. Second, caregiving touch, such as warming an infant, regulates affect by socially enacting homeostatic control and co-regulation of physiological states. Third, affective touch such as gentle stroking or tickling regulates affect by allostatic regulation of the salience and epistemic gain of particular experiences in given contexts and timescales. These three functions of affective touch are most likely mediated, at least partly, by different neurobiological processes, including convergent hedonic, dopaminergic and analgesic, opioidergic pathways for the attachment function, 'calming' autonomic and endocrine pathways for the homeostatic function, while the allostatic function may be mediated by oxytocin release and related 'salience' neuromodulators and circuits.

\footnotetext{
Addresses

${ }^{1}$ Research Department of Clinical, Educational and Health Psychology, University College London, London, UK

${ }^{2}$ Department of Psychology, Royal Holloway University of London, London, UK

${ }^{3}$ Department of Primary Care and Mental Health, University of Liverpool, Liverpool, UK
}

Corresponding author: Fotopoulou, Aikaterini (a.fotopoulou@ucl.ac.uk)

\section{Current Opinion in Behavioral Sciences 2021, 44:80-87}

This review comes from a themed issue on Body-brain interactions/ affective touch folks

Edited by Annett Schirmer and Francis McGlone

https://doi.org/10.1016/j.cobeha.2021.08.008

2352-1546/@ 2021 The Authors. Published by Elsevier Ltd. This is an open access article under the CC BY-NC-ND license (http://creativecommons.org/licenses/by-nc-nd/4.0/).

\section{Introduction: self versus social affective regulation}

Affective regulation refers to the monitoring and modulation of the intensity, duration or nature of an affective state in pursuit of homeostasis (e.g. Ref. [1]). Affective states are typically valenced (positive or negative) feelings of variable arousal with corresponding behavioural tendencies of approach or avoidance. Some authors distinguish between affect and emotion regulation. Here, we use the term affect regulation more generally to describe the regulation of any affective state, whether or not it is occurring within a more specific emotional experience. For example, anger, fear, anxiety, and pain can all be said to be accompanied by negative affect that one may be motivated to reduce.

Affective regulation has been described primarily in intrapersonal terms as the ability to exercise cognitive control over one's affective states (self-regulation; [2]), either before (e.g. avoiding a stressful situation) or after affect is experienced (e.g. suppressing one's outward expression of inner feelings; [1]). The majority of this work has focused on higher-order cognitive abilities and their neural correlates, such as the role of prefrontal systems in modulating the activity of subcortical systems [3] and related neuroendocrinological pathways, including the downregulation of the hypothalamus-pituitaryadrenocortical (HPA) system during stressful situations [4]. However, there are also parallel traditions in animal research, psychology, and more recently social cognitive neuroscience, that have understood the role of interpersonal and social mechanisms in affect regulation [5-7]. Importantly, these literatures have understood that affect can be modulated by both cognitive processes and embodied processes, such as social touch $\left[8,9^{\circ}, 10\right]$. Embodied forms of social, affective regulation are defined here as social interactions involving the body that do not include explicit, verbal, or symbolic communications. More broadly, a cognitive process is defined as embodied if it is at least partly constituted by bodily processes beyond the brain.

Yet, an integrative theoretical framework for understanding both embodied and cognitive effects of social regulation is still missing [11,12]. Characteristically, one of the most influential recent theories of social emotion regulation, Social Baseline Theory, states that the brain's default expectation is the availability of social resources and thus the resource-demanding task of regulation can be outsourced to others [13]. While we agree with this perspective [14], we argue that the social regulation of affect entails more embodied and cognitive effects than mere signaling of social presence and protection.

Specifically, we focus on social touch as a paradigmatic case of socioaffective regulation, and put forward a model of the regulatory mechanisms involved, ranging from embodied modulation to metacognitive monitoring. We 


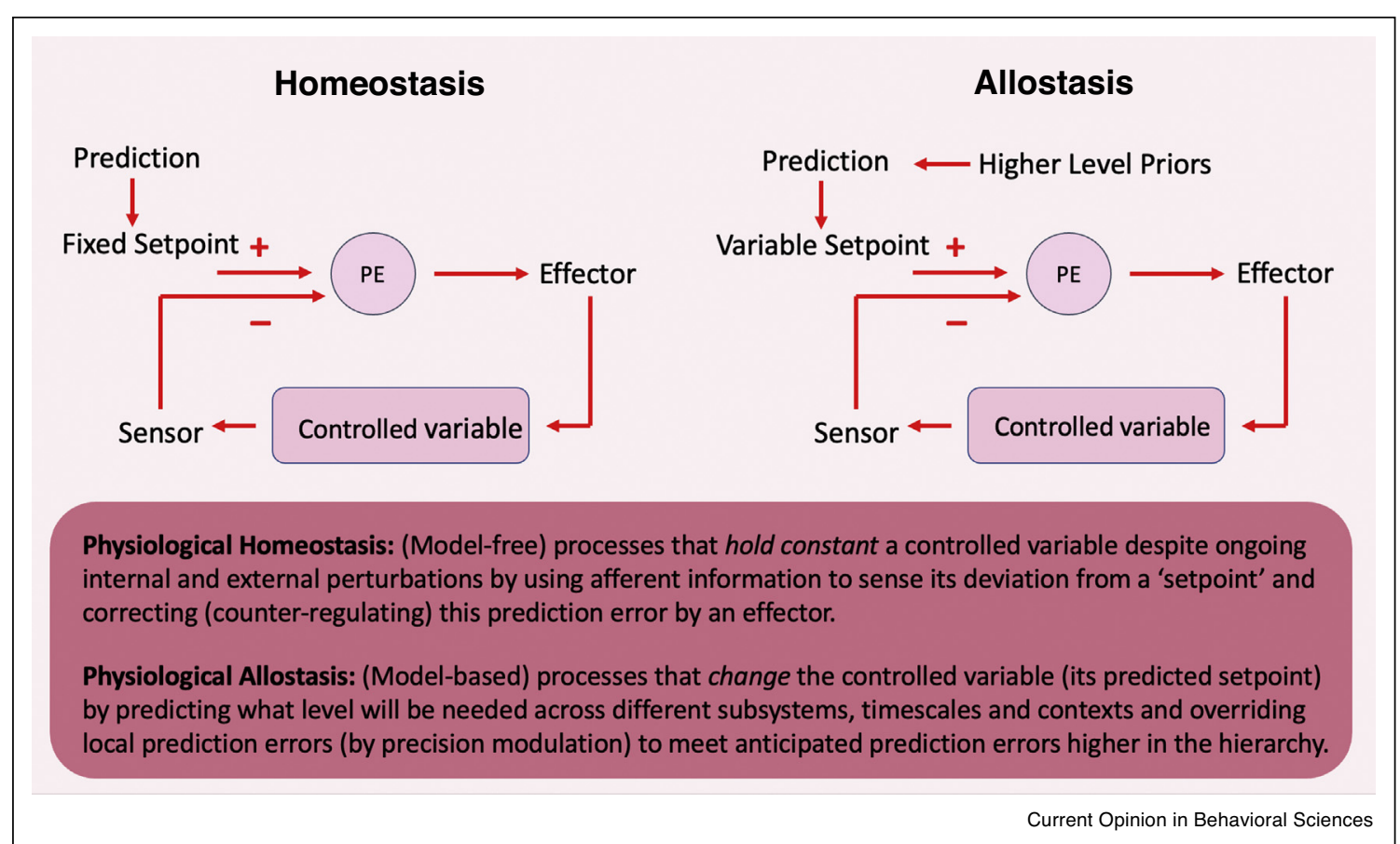

A schematic representation of differences and similarities between homeostatic and allostatic regulation.

Although the exact relation between the terms homeostasis and allostasis remains debated, we follow certain active inference theories in regarding allostasis as an extension of direct homeostatic control to flexible, indirect control via 'counterfactual' predictions about future interoceptive states [29]. Left panel: Using afferent information to sense homeostatic deviation, processes are used to match fixed homeostatic setpoints by correcting (i.e. counter-regulating) any detected errors. Right panel: By predicting what level will be needed to change or delay a variable setpoint (according to contextual demands and long-term goals), processes are used to override local prediction errors in the service of anticipated prediction errors higher in the hierarchy [29].

envision that such processes converge in affective regulation but we emphasise both embodied cognition and higher-order concepts of cognition in this paper to ensure coverage of the whole spectrum of functions. This model is embedded in wider perspectives on the central role of homeostasis, rather than rationality, in cognition, and particularly the theoretical framework 'Mentalising Homeostasis' that we have proposed to characterise the interdependence between interoception, cognition and socialisation $\left[12,14-16,17^{\circ}\right]$. According to this widely discussed framework (see commentaries on $\left[12,18,19^{\circ}\right]$ ), affect is not the result of ascending 'sensory' signals and descending 'modulatory' cognitive influences, but rather the result of a single hierarchical system of recurring processes including predictive feedforward and feedback loops (e.g. [20]), as understood particularly in hierarchical Bayesian brain accounts [21]. We review recent literature from the perspective of this framework to offer a unifying account on the embodied, cognitive and metacognitive processes by which tactile, social interactions regulate affect.

\section{Contact comfort: social proximity and attachment as a first prior}

We propose that maternal contact and early touch with caregivers in infancy constitute a first prior $\left[17^{\circ}\right]$, or an evolutionary prescribed prediction for contact comfort [22] and social attachment [23]. This prior is fulfilled in early development with corresponding physiological mechanisms for seeking and enjoying (dopaminergic and opioidergic pathways, respectively [24]) skin-to-skin contact and social proximity, and avoiding social isolation. These mechanisms are assumed to have affective correlates, so that social contact is accompanied by positive, hedonic affect and corresponding approach tendencies, while social isolation is experienced as distressing and leads to corresponding avoidance tendencies. Indeed, variation in postnatal (skin-to-skin) touch regulates several physiological dimensions, such as fear, noxious and stress reactivity $\left[25^{\circ}, 26^{\bullet}, 11\right]$, including physiological, epigenetic and neuroendocrine processes involved in the development of adaptive HPA reactivity [27]. The primary, 'baseline' [13] function of such 'contact comfort' 
Figure 2

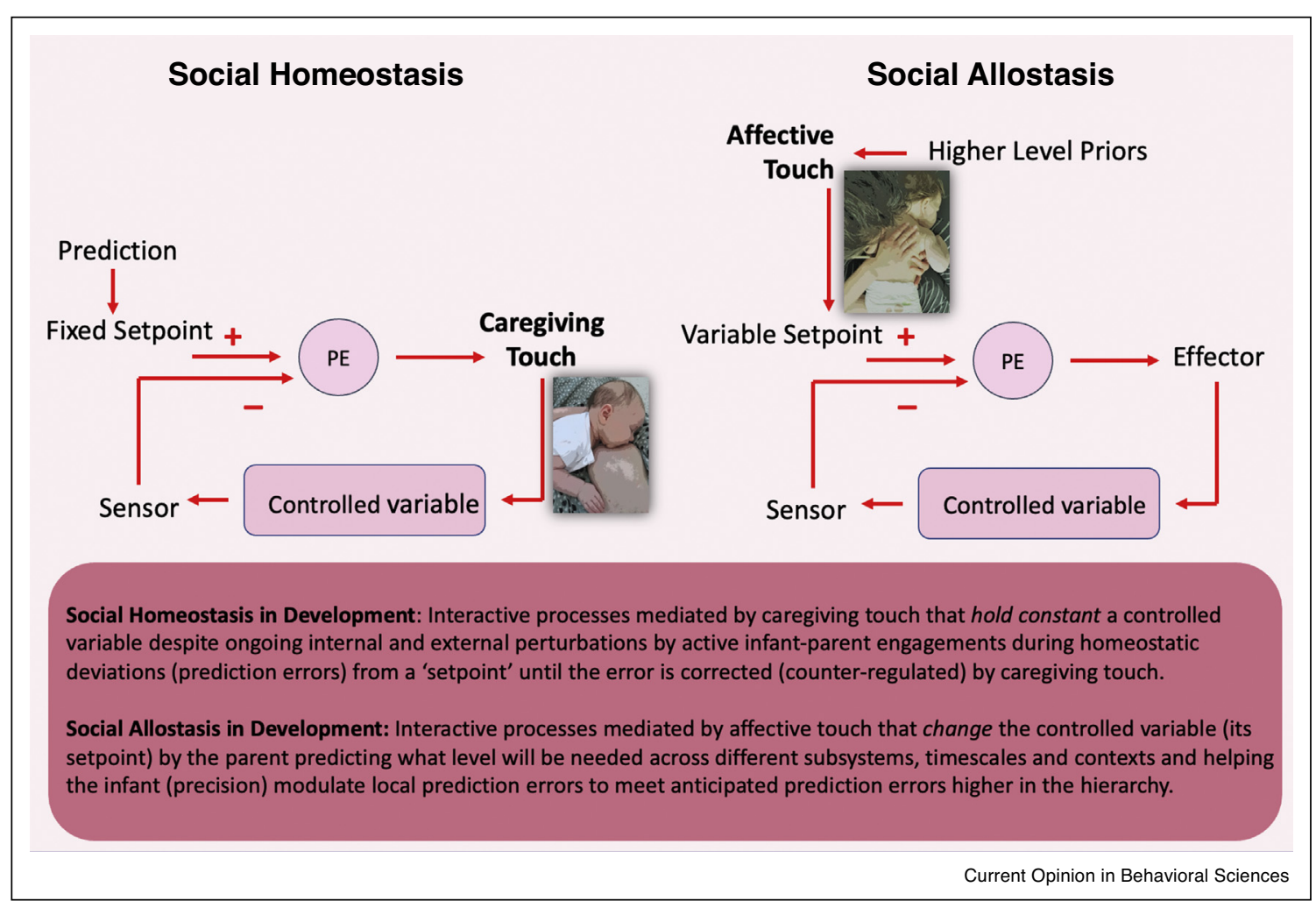

A schematic representation of our model sinthesizing the role of touch in social homeostasis and allostasis in development.

Left panel: Caregiving touch serves to match fixed homeostatic setpoints by correcting (i.e. counter-regulating) any detected errors. Such interactions are needed given that the unaided infant cannot act to correct (counter-regulate) interoceptive error signals and hence close the interoceptive action-perception loop by herself. Right panel: Affective touch in the dyad is used to change or delay a variable setpoint according to contextual demands and long-term goals. By doing this, the parent helps the infant tolerate local prediction errors in the service of anticipated prediction errors higher in the hierarchy.

mechanisms is typically understood as the increase of the infant's engagement with the caregiver, ultimately promoting social attachment as a safe base for cognitive, affective and physical development $\left[28^{\circ}\right]$ and a life-long need for social affiliation.

However, as social and embodied regulation are highly integrated from the outset, we propose that the role of touch in development and cognition is not limited to this primary attachment function, but rather it extends to at least two further functions, namely homeostatic regulation [12] and allostatic regulation ([6,18]; see Figure 1 for definitions). These functions are, of course, tightly interlinked but for clarity we outline them here in turn. Ultimately, all of these touch functions serve homeostasis, they overlap in everyday life, and we are not assuming here that any is more primary than the rest. For example, a father may hold an infant affectively to feed and calm them, while also fulfilling attachment needs. Our aim is rather to highlight how each of these touch functions, in isolation or in combination, contribute to affect regulation.

\section{Caregiving touch as homeostatic affect regulation}

First, at a homeostatic level, touch involved in everyday caregiving, typically considered as merely 'instrumental' or 'utilitarian' (defined as touch aimed primarily at practical goal attainment such as feeding, holding, cleaning and so on rather than affective or hedonic effects such as calming or stimulating), can play a unique role in interoceptive inference and what we have called the 'embodied mentalisation' of interoception [12]. When a parent feeds, or holds an infant, they are not just maintaining attachment and informing the infant about her social mileau; they are also simultaneously actively modulating the infant's physiological states, her internal mileau, and hence her affect. Given the prolonged motor immaturity of human infants, proximal engagement with caregivers is necessary for infants to regulate homeostasis and 
eventually build generative models regarding their bodily needs (e.g. plan the actions that would lead to satiation when hungry). Otherwise, the unaided infant cannot fulfill their interoceptive predictions. Thus, most interoceptive regulation in infancy is performed via caregiving touch (see Figure 2). For instance, human newborns are unable to maintain their body temperature without thermal protection. Signals from thermoreceptors reach the hypothalamus, leading to norepinephrine release that then triggers nonshivering thermogenesis, or lipolysis of brown adipose tissue [30]. This main homeothermic heat production mechanism is insufficient in newborns because it depends on the levels of available adiposity and certain enzymes that only build up later in development. Instead, a more effective mechanism is infant affective behavior, for example, prompting caregiving by crying. It follows that the rate by which bodily interactions between caregivers and infants fulfill interoceptive predictions is accompanied by arousal reduction, motor relaxation [31], and affect modulation (prediction error rate reduction leading to positive affect and vice versa; [32]). Thus, caregiving touch leads to arousal and valence fluctuations regarding homeostatic needs, and can regulate affect by the corresponding autonomic and endocrine pathways, in addition to the aforementioned hedonic, calming and analgesic benefits of the touch itself.

More generally, during these proximal interactions in early infancy, caregivers offer multimodal 'matching' between their bodies and those of their infants [33]. Studies in cardiorespiratory synchronization, for example, show that touch can entail an embodied transfer of the carer's own parasympathetic regulation to the infant [34]. Moreover, a mother's hug that has somatosensory, cardiorespiratory and thermoregulation consequences is accompanied also by her smell, her song and her face [ $\left.35^{\circ}\right]$. Multisensory input about the maternal body is thus bound together in common inferences about the causes of changes to one's physiological states (embodied mentalisation; [12]), with this binding possibly being processed by the hippocampus even before episodic memories can be laid down [36]. In everyday life, feeding, sleeping, bathing routines typically include endless repetitions of multisensory and affect-modulating bundles from at least two bodies. Thus, touch is a central building block of the mentalization of the internal milieu of the body, that is, the progressive build-up of multisensory, autonomic, and motor predictions about the variable physiological states of the body and its couplings with the outside world. Note that the term mentalization is used here according to the above descriptions, further specified in Ref. [12], to denote probabilistic, inferential processes built on the basis of frequently encountered multisensory input. We and other authors have claimed (see Ref. [12] for review) that this more specific use of the term is useful as it can highlight the continuity of these
Bayesian inferences, from multisensory integration to social cognition. Finally, although there are conceptual and empirical gaps between research in childhood and in adulthood, interpersonal and group synchrony in adulthood has also been suggested to have regulatory effects on affect, particularly in studies on empathy, collaboration, and joint action (e.g. Ref. [37 $\left.7^{\circ}\right)$.

\section{Affective touch as allostatic affect regulation}

The second way in which social touch contributes to affect regulation relates to allostasis. Allostasis refers to achieving physiological stability through preemptive change across different temporal scales, for example adjusting one's metabolic needs in certain environments where foraging is dangerous ([38]; see Figure 1). According to certain theories of allostasis [39], allostatic regulation relies on learned, hierarchically organized, generative models of how interoceptive-exteroceptive couplings are likely to change over time. More specifically, while homeostatic control enslaves reflexes to produce actions (effectors in Figure 1) that correct prediction errors and fulfill beliefs about bodily states, allostasis requires a temporary change or suspension of interoceptive set points, effectively altering the priors (beliefs) of the relevant homeostatic reflex arc under the guidance of higher predictive models about future perturbations of bodily states.

We propose that certain types of social touch, such as stroking or tickling, that have a communicative role in early social interactions [8] and across the lifespan [40] are implicated in allostatic control (Figure 2). Specifically, the role of affective, communicative touch is not only to match some fixed homeostatic setpoint as in the case of caregiving touch, but frequently this touch can be used to delay or change a variable setpoint according to contextual demands and long-term goals (Figure 2). While, as aforementioned, attachment-promoting and caregiving touch aims to satisfy the infant's social and bodily needs in a contingent, synchronous way [33], affective touch appears to promote a rebalancing between homeostatic and allostatic needs in a given bodily and social context. For example, a father may tickle an infant to keep them awake a bit longer to better regulate their sleeping-eating cycle around the mother's availability. Indeed, there is evidence that stroking an infant can act as an embodied, ostensive cue directing attention towards expected epistemic gains, such as learning the identity of faces $\left[41^{\circ}\right]$. Epistemic gains are defined as increased opportunities for new knowledge acquisition. In adults, socioaffective touch can modulate the salience of noxious stimulation in given contexts and related responses in anterior insular and cingulate cortices $[15,16]$. Salience is defined as the relative property of a stimulus or state that makes it stand out from its immediate context, and it is considered of key importance during learning as it allows organisms to focus their limited resources on pertinent stimuli or states in 


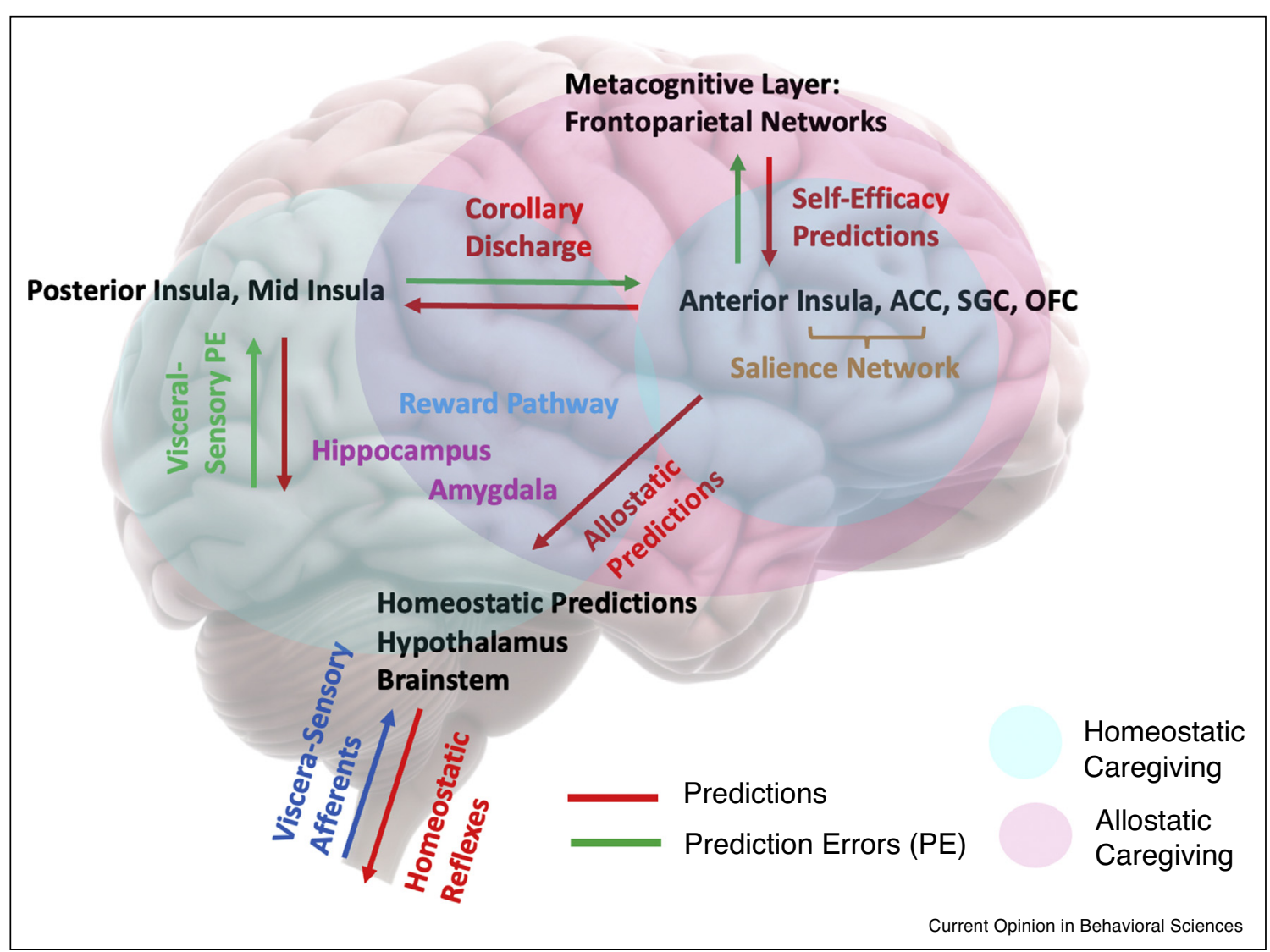

A proposed circuit for interoception, allostatic and metacognitive regulation of homeostatic reflex arcs, and the role of caregiving and affective touch.

Blue lines: sensory inputs; red lines: predictions; green lines: prediction errors (PE). In this model, we expand on the allostatic regulation model previously proposed by [39] by introducing novel aspects such as homeostatic and allostatic caregiving. Here, regions such as the anterior insula ( $\mathrm{AIC}$ ), anterior cingulate cortex (ACC), orbitofrontal cortex (OFC), and subgenual cortex (SGC) are at the top of this circuit (i.e. generating allostatic predictions, as they embody a generative model of inputs in order to infer current bodily states but also predict future states). These areas have access to prediction errors about interoception arriving from the posterior and mid-insula, as well as connectivity that conveys allostatic predictions and allows them to modulate homeostatic beliefs in a collection of subcortical regions, including the central nucleus of the amygdala, the ventral and dorsal striatum, fulfilled by reflex arcs in regions like the hypothalamus or brainstem. Critically, descending projections from the AIC, ACC, OFC, and SGC could send the same prediction to posterior and mid-insula serving as a corollary discharge against which sensory inputs can be compared, with the resulting PEs returned to the AIC, ACC, OFC, and SGC for allostatic adjustments. PEs from these brain areas can also project to frontoparietal networks, which in turn send descending self-efficacy predictions and ultimately influence allostatic predictions. With respect to homeostatic caregiving (shaded in blue), we propose that the main areas correspond to the amygdala, hippocampus, posterior and mid-insula, and salience networks (primarily the AIC and ACC, in connection with the tempoparietal junction), with its regulation implicating endogenous opioids and dopamine. In contrast, for allostatic caregiving (shaded in pink), we propose that the main areas are the AIC, ACC, OFC, and SGC (deepest level of a wider circuit for allostasis), with this kind of top-down prediction mediated by neuromodulatory mechanisms (e.g. cholinergic and dopaminergic in fronto-striatal circuits) and neuropeptides (e.g. oxytocin for social contexts). We further propose that allostatic caregiving engages the amygdala, hippocampus and salience networks, with their connections to frontoparietal networks serving 'metacognition' or precision optimization purposes.

given environments. Thus, social touch may be a way by which humans not only outsource homeostatic regulation to caregivers (via caregiving touch; see preceding section), but also outsource the processing of salience and epistemic gain to their social environment (via affective touch), so as best to learn to eventually serve their own allostasis in a given social and physical niche $\left[42^{\circ}, 43\right]$.
The neurobiology of allostasis cannot fully be covered here, but existing theories (e.g. Ref. [39]) converge on the role of anterior insular and cingulate cortex (AIC and ACC), subgenual and orbitofrontal cortex (SGC and OFC), as comprising the deepest level of a wider circuit for allostasis (see Figure 3). These areas have access to prediction errors about interoception arriving in granular 
layer IV of the primary interoceptive insular cortex, and the connectivity that allows them to modulate homeostatic beliefs in a collection of subcortical regions, including the central nucleus of the amygdala, the ventral and dorsal striatum, fulfilled by reflex arcs in regions like the hypothalamus or brainstem [39]. Importantly, such topdown predictions do not only represent the content of lower-level representations but also predict their uncertainty in given contexts, defined in the above models as the precision of a probability distribution (inverse variance or uncertainty). This kind of top-down prediction in sensory cortices is thought to be mediated by neuromodulatory mechanisms that optimize the attentional gain of populations encoding prediction errors, such as cholinergic and dopaminergic modulations in fronto-striatal circuits [44], and by neuropeptides such as oxytocin in social contexts [45]. In interoception, precision may relate to interoceptive sensitivity [46] and may be modulated by contextual factors at different allostatic scales. Of relevance, the aforementioned central hubs of allostatic control, namely AIC and ACC, in connection with temporoparietal junction regions, form part of the salience network, with a recognised role in modulating the salience of prediction errors during interoception, multisensory integration, and body awareness [47].

In addition, these areas, together with OFC, SGC and amygdala are considered the key target areas of central oxytocinergic modulations, as well as central hubs of brain circuits supporting affective touch, and particularly stroking touch [48], activating a population of unmyelinated Ctactile (CT) afferents [50]. This system, associated with oxytocin release in both animals (e.g. Ref. $\left[49^{\circ}\right]$ ) and humans (e.g. Ref. [50]), is thought to play a fundamental but underspecified social role. Somewhat similarly, the social role of oxytocin is increasingly understood in allostatic terms $\left[5^{\circ}\right]$. Indeed, oxytocin may exert effects on interoception by modulating the salience of sensory stimuli in relation to the social context in which they occur [15]. Thus, affective touch, via its relation to oxytocin and other neuromodulators, can act as a key precision modulator (e.g. Refs. [15,16,45]) during social allostasis, modulating key homeostatic setpoints according to long-term needs.

Finally, we follow recent models of interoceptive inference $\left[52^{\circ}, 39\right]$ to speculate that the role of affective touch in allostatic regulation has implications for metacognition. Specifically, the degree to which the developmental history of affective touch provision affects the development of allostatic regulation will form part of particular metacognitive styles about affect regulation (e.g. interoceptive confidence-accuracy relations, self-efficacy beliefs, expectations about the trustworthiness of others during threat, or attachment styles; Figure 3; [12,39,43]). For example, attachment styles have also been found to relate not only to the perception of affective touch [53], but also to the degree to which pain is modulated by affective touch [54]. Further, the degree to which intranasal oxytocin downregulates HPA reactivity is related to individual differenes in affective regulation, with the authors proposing that appropriate, oxytocinergic parental care in childhood, like affective touch interactions, may have optimized the use of oxytocin for dealing with stressors in adulthood with the appropriate metacognitive strategies [55]. More generally, metacognitive traits about the controllability of sensory states as well as established attachment schemas have been linked with different neuroendocrinological and neural responses to stress [55]. This proposal is also based on numerous reports of how interoceptive states can directly influence uncertainty in both interoceptive and non-interoceptive domains and hence impinge on metacognitive judgements (e.g. Ref. [56]), but the specific development and life-long relationship between affective touch and metacognitive control will need to be tested and specified in future studies.

\section{Conclusion}

Typically, affect regulation is studied within two different traditions: self-regulation as a form of cognitive control and social regulation as a form of social support. In this paper, we offer a unifying perspective of these literatures. Specifically, we described social touch as a paradigmatic case of social, affective regulation of both positive and negative affect, and we have argued that the role of touch in development and cognition, and particularly affect regulation, is not limited to attachment and social protection. Instead, social touch seems to contribute to affective regulation with functions that range from direct, physiological co-regulation to the development of allostatic, cognitive, and metacognitive models of regulation and social cognition. Specifically, social touch informs us about our social milieu, as it simultaneously regulates our internal milieu, and more broadly our homeostasis. Caregiving touch, in particular, plays a unique role in adaptive interoceptive inference, that is, the progressive build-up of multisensory, autonomic and motor predictions about the variable physiological states of the body and its couplings with the outside world. Finally, affective touch contributes to social allostasis, or predictive homeostasis. Thus, humans, starting in infancy, seem to outsource some of the regulation of both the early attachment and homeostatic needs to caregivers and the processing of salience and epistemic gain, so as best to learn to eventually serve their own allostasis in a given social, physical, and cultural environment.

\section{Authors' contributions}

A.F. wrote the manuscript with all other authors providing critical additions, sections and revisions. All authors approved the final version of the manuscript for submission. 


\section{Conflict of interest statement}

Nothing declared.

\section{Acknowledgement}

This work was supported by a European Research Council Consolidator Award [ERC-2018-COG-818070] (to AF).

\section{References and recommended reading}

Papers of particular interest, published within the period of review, have been highlighted as:

- of special interest

1. Gross JJ: Emotion regulation: conceptual and empirical foundations. Handbook of Emotion Regulation. edn 2. New York, NY, US: The Guilford Press; 2014, 3-20.

2. Bandura A et al.: Role of affective self-regulatory efficacy in diverse spheres of psychosocial functioning. Child Dev 2003, 74:769-782.

3. Buhle JT et al.: Cognitive reappraisal of emotion: a metaanalysis of human neuroimaging studies. Cereb Cortex 2014, 24:2981-2990.

4. Kudielka BM, Hellhammer DH, Wüst S: Why do we respond so differently? Reviewing determinants of human salivary cortisol responses to challenge. Psychoneuroendocrinology 2009, 34:2-18.

5. Sbarra DA, Hazan C: Coregulation, dysregulation, selfregulation: an integrative analysis and empirical agenda for understanding adult attachment, separation, loss, and recovery. Pers Soc Psychol Rev 2008, 12:141-167.

6. Schulkin J: Social allostasis: anticipatory regulation of the internal milieu. Front Evol Neurosci 2011, 2:111.

7. Reeck C, Ames DR, Ochsner KN: The social regulation of emotion: an integrative, cross-disciplinary model. Trends Cogn Sci 2016, 20:47-63.

8. Hertenstein MJ: Touch: its communicative functions in infancy. Hum Dev 2002, 45:70-94.

9. Field T: Social touch, CT touch and massage therapy: a - $\quad$ narrative review. Devel Rev 2019, 51:123-145

This review brings together evidence from multiple research traditions on the role of different forms of touch in development and wellbeing. A usefu starting point for delving into literature on effects of touch on stress and pain, and of touch on development across the lifespan.

10. von Mohr M et al.: The social buffering of pain by affective touch: a laser-evoked potential study in romantic couples. Soc Cogn Affect Neurosci 2018, 13:1121-1130.

11. Morrison I: Keep calm and cuddle on: social touch as a stress buffer. Adapt Hum Behav Physiol 2016, 2:344-362.

12. Fotopoulou A, Tsakiris M: Mentalizing homeostasis: the social origins of interoceptive inference. Neuropsychoanalysis 2017, 19:3-28.

13. Beckes L, Coan JA: Social baseline theory: the role of social proximity in emotion and economy of action. Soc Personal Psychol Compass 2011, 5:976-988.

14. Decety J, Fotopoulou A: Why empathy has a beneficial impact on others in medicine: unifying theories. Front Behav Neurosci 2015, 8.

15. Krahé $\mathrm{C}$ et al.: The social modulation of pain: others as predictive signals of salience - a systematic review. Front Hum Neurosci 2013, 7:386.

16. von Mohr M, Fotopoulou A: The cutaneous borders of interoception: active and social inference of pain and pleasure on. The Interoceptive mind: From Homeostasis to Awareness 2018, vol 102.

17. Ciaunica A et al.: The first prior: from co-embodiment to co-

- homeostasis in early life. Conscious Cogn 2021, 91:103117
This interdisciplinary perspective papers emphasises that humans build their first, predictive models of homeostatic control inside the womb, coregulated by the mother. The paper describes the implications of this coembodiment and co-homeostasis for cognition.

18. Atzil S et al:: Growing a social brain. Nat Hum Behav 2018:1

19. Burleson MH, Quigley KS: Social interoception and social - allostasis through touch: legacy of the somatovisceral afference model of emotion. Soc Neurosci 2021, 16:92-102.

20. Siegel M, Körding KP, König P: Integrating top-down and bottom-up sensory processing by somato-dendritic interactions. J Comput Neurosci 2000, 8:161-173.

21. Friston K: The free-energy principle: a unified brain theory? Nat Rev Neurosci 2010, 11:127-138.

22. Harlow HF, Zimmermann RR: Affectional responses in the infant monkey. Science 1959, 130:421-432.

23. Bowlby J: Attachment and Loss, vol 1: Attachment. 1997/1969, London: Random House.

24. Panksepp J: Affective Neuroscience: The Foundations of Human and Animal Emotions. USA: Oxford University Press; 1998.

25. Manzotti A et al.: Dynamic touch reduces physiological arousal - $\quad$ in preterm infants: a role for c-tactile afferents? Dev Cogn Neurosci 2019, 39:100703.

26. Cascio CJ, Moore D, McGlone F: Social touch and human - development. Dev Cogn Neurosci 2019, 35:5-11

This extensive review considers the role of social touch healthy and disordered development, as to some degree across the life span, considering both bottom up afferent systems and top-down factors such as culture and contextual influences.

27. Meaney MJ: Maternal care, gene expression, and the transmission of individual differences in stress reactivity across generations. Ann Rev Neurosci 2001, 24:1161-1192.

28. Norholt $\mathrm{H}$ : Revisiting the roots of attachment: a review of the

- biological and psychological effects of maternal skin-to-skin contact and carrying of full-term infants. Infant Behav Dev 2020 60:101441.

29. Corcoran AW, Pezzulo G, Hohwy J: From allostatic agents to counterfactual cognisers: active inference, biological regulation, and the origins of cognition. Biol Philos 2020, 35:145.

30. IJzerman $\mathrm{H}$ et al.: A theory of social thermoregulation in human primates. Front Psychol 2015, 6.

31. Esposito G et al:: Infant calming responses during maternal carrying in humans and mice. Curr Biol 2013, 23:739-745.

32. Joffily M, Coricelli G: Emotional valence and the free-energy principle. PLOS Comput Biol 2013, 9:e1003094.

33. Feldman R: Parent-infant synchrony and the construction of shared timing; physiological precursors, developmental outcomes, and risk conditions. J Child Psychol Psychiatry 2007 48:329-354.

34. Van Puyvelde $\mathrm{M}$ et al.: Infants autonomic cardio-respiratory responses to nurturing stroking touch delivered by the mother or the father. Front Physiol 2019, 10:1117.

35. Rekow D et al.: Odor-driven face-like categorization in the - $\quad$ human infant brain. Proce Natl Acad Sci U S A 2021, 118.

36. Ellis CT et al: : Evidence of hippocampal learning in human infants. Curr Biol 2021.

37. Korisky A et al: : A dual-brain approach for understanding the

- neuralmechanisms that underlie the comforting effects of social touch. Cortex 2020, 127:333-346

This study implements a dual brain approach with $\mathrm{FMRI}$, that is, it scans two people consecutively while they share an experience, in this instance handholding during pain. The study confirms the hypothesis that social touch involves similar activations between the suffering 'target' and the empathizer in brain regions related to emotional sharing such as the observation-execution (mirror) network in the inferior parietal lobule. 
38. Sterling P, Eyer J: Allostasis: a new paradigm to explain arousal pathology. Handbook of Life Stress, Cognition and Health. Oxford, England: John Wiley \& Sons; 1988, 629-649.

39. Stephan KE et al.: Allostatic self-efficacy: a metacognitive theory of dyshomeostasis-induced fatigue and depression. Front Hum Neurosci 2016, 10.

40. Kirsch LP et al.: Reading the mind in the touch: neurophysiological specificity in the communication of emotions by touch. Neuropsychologia 2018, 116:136-149.

41. Della Longa L, Gliga T, Farroni T: Tune to touch: affective touch - enhances learning of face identity in 4-month-old infants. Devel Cogn Neurosci 2019, 35:42-46

Ostensive cues, such as direct gaze enhance social information processing. This well-conducted psychophysical study shows that affective touch may also act as a specific, ostensive cue, promoting better processing of concurrent social information, already in 4-month old infants.

42. Theriault JE, Young L, Barrett LF: The sense of should: a

- biologically-based framework for modeling social pressure. Phys Life Rev 2021, 36:100-136

This innovative article proposes a novel basis for understanding the experience of social pressure, the Sense of Should (SoS) not as inflicted by external punishment, but rather as the internalised need to align oneself to others' expectations in order to promote stability in one's social environment and thus ultimately optimise the expenditure of one's metabolic re-sources.

43. Fotopoulou A: Mentalising allostasis: the sense that I should eat. Comment on" The sense of should: a biologically-based framework for modeling social pressure" by Jordan E. Theriault, Liane Young, and Lisa Feldman Barrett. Phys Life Rev 2021, 36:20-23.

44. Cox J, Witten IB: Striatal circuits for reward learning and decision-making. Nat Rev Neurosci 2019, 20:482-494.

45. Crucianelli $L$ et al:: Embodied precision: intranasal oxytocin modulates multisensory integration. J Cogn Neurosci 2018:115 .

46. Ainley V et al: 'Bodily precision': a predictive coding account of individual differences in interoceptive accuracy. Philos Trans $R$ Soc B: Biol Sci 2016, 371:20160003.
47. Kirsch LP et al.: Updating beliefs beyond the here-and-now: the counter-factual self in anosognosia for hemiplegia. Brain Commun 2021, 3:fcab098 http://dx.doi.org/10.1093/braincomms/ fcab098.

48. Morrison I: ALE meta-analysis reveals dissociable networks for affective and discriminative aspects of touch. Hum Brain Mapp 2016, 37:1308-1320.

49. Tang $Y$ et al: : Social touch promotes interfemale

- communication via activation of parvocellular oxytocin neurons. Nat Neurosci 2020, 23:1125-1137.

50. Grewen KM et al.: Effects of partner support on resting oxytocin, cortisol, norepinephrine, and blood pressure before and after warm partner contact. Psychosom Med 2005, 67:531538.

51. Quintana DS, Guastella AJ: An allostatic theory of oxytocin. Trends Cogn Sci 2020, 24:515-528

This article presents an allostatic theory of oxytocin, proposing that the oxytocin system evolved to - and continues to support - allostatic regulation. The wide range of social and non-social processes influenced by oxytocin through both peripheral and central mechanisms is viewed through the lens of promoting allostasis.

52. Allen M: Unravelling the neurobiology of interoceptive

- inference. Trends Cogn Sci 2020, 24:265-266 http://dx.doi.org/ 10.1016/j.tics.2020.02.002.

53. Krahé $\mathrm{C}$ et al:: Sensitivity to CT-optimal, affective touch depends on adult attachment style. Scientific reports 2018, 8:14544.

54. Krahé $\mathrm{C}$ et al.: Affective touch and attachment style modulate pain: a laser-evoked potentials study. Philos Trans $R$ Soc $B$ 2016, 371:20160009.

55. Quirin M, Kuhl J, Düsing R: Oxytocin buffers cortisol responses to stress in individuals with impaired emotion regulation abilities. Psychoneuroendocrinology 2011, 36:898-904.

56. Allen $\mathrm{M}$ et al.: Unexpected arousal modulates the influence of sensory noise on confidence. eLife 2016, 5:e18103. 